\title{
Angular Spectrum Simulation of Pulsed Ultrasound Fields
}

\author{
Du, Yigang; Jensen, Henrik; Jensen, Jørgen Arendt
}

Published in:

2009 IEEE International Ultrasonics Symposium (IUS)

Link to article, DOI:

10.1109/ULTSYM.2009.5441670

Publication date:

2009

Document Version

Publisher's PDF, also known as Version of record

Link back to DTU Orbit

Citation (APA):

Du, Y., Jensen, H., \& Jensen, J. A. (2009). Angular Spectrum Simulation of Pulsed Ultrasound Fields. In 2009 IEEE International Ultrasonics Symposium (IUS) (pp. 2379-2382). IEEE.

https://doi.org/10.1109/ULTSYM.2009.5441670

\section{General rights}

Copyright and moral rights for the publications made accessible in the public portal are retained by the authors and/or other copyright owners and it is a condition of accessing publications that users recognise and abide by the legal requirements associated with these rights.

- Users may download and print one copy of any publication from the public portal for the purpose of private study or research.

- You may not further distribute the material or use it for any profit-making activity or commercial gain

- You may freely distribute the URL identifying the publication in the public portal

If you believe that this document breaches copyright please contact us providing details, and we will remove access to the work immediately and investigate your claim 


\title{
Angular Spectrum Simulation of Pulsed Ultrasound Fields
}

\author{
Yigang $\mathrm{Du}^{1,2}$, Henrik Jensen ${ }^{2}$ and Jørgen Arendt Jensen ${ }^{1}$ \\ 1) Center for Fast Ultrasound Imaging, DTU•Elektro, Bldg. 349, \\ Technical University of Denmark, DK-2800 Kgs. Lyngby, Denmark. \\ 2) BK Medical Aps, Mileparken 34, DK-2730 Herlev, Denmark.
}

\begin{abstract}
The optimization of non-linear ultrasound imaging should in a first step be based on simulation, as this makes parameter studies considerably easier than making transducer prototypes. Such a simulation program should be capable of simulating non-linear pulsed fields for arbitrary transducer geometries for any kind of focusing and apodization. The Angular Spectrum Approach (ASA) is capable of simulating monochromatic non-linear acoustic wave propagation. However, for ultrasound imaging the time response of each specific point in space is required, and a pulsed ASA simulation with multi temporal frequencies must be performed. Combining it with Field II, the generation of non-linear simulation for any geometry with any excitation array transducer becomes feasible. The purpose of this paper is to make a general pulsed simulation software using the modified ASA. Linear and phased array transducers are used to create the source plane, which is $\mathbf{2} \mathbf{~ m m}$ from the transducer surface. Field II generates pulses for all the points in the source plane, and the 3D matrix data (1D time, 2D space) are obtained. The pulses in the simulated plane are calculated by the modified ASA, which is the 3D inverse Fourier transform of the values in a series of planes corresponding to each temporal frequency. The values in the planes are the multiplications between the 2D spatial Fourier transform of the pressure in the source plane and the ASA propagator for every temporal frequency components. The beam focusing is produced by Field II in the source plane. A rectangular plane matched to the shape of the transducer surface is chosen as the source. The plane covering $12.7 \times 156.3 \mathrm{~mm}^{2}$ has $33 \times 407$ points with a spatial sampling interval of $1 / 2$ wavelength. A comparison of ASA to Field II at the focal point $(0,0,64) \mathrm{mm}$ for a 64 -element, $2 \mathrm{MHz}$ linear array transducer has been made in the paper, and the root mean square (RMS) error is $2.7 \%$. For further validation, 3 randomly selected points in the simulated plane have RMS errors of $12.5 \%, 13.3 \%, 23.4 \%$ at the positions (3.9, $-1.5,64),(-1.9,1.9,64),(6.2,-4.2,64) \mathrm{mm}$. The RMS error of the pulses for all points in the simulated plane is $10.9 \%$. The good agreement between ASA and Field II simulation for the pulsed ultrasound fields obtained in this paper makes it possible to expand Field II to non-linear pulsed fields.
\end{abstract}

\section{INTRODUCTION}

Currently, many programs for simulating the ultrasound beam and propagation have been made. These can be found from the IEEE UFFC official website www.ieee-uffc.org/ultrasonics/software.asp. There are a lot of different types of softwares using different methods for both linear [1] [2] and non-linear [3] [4] acoustic field simulation. However, those are not capable of providing enough functions for the transducers settings to simulate non-linear ultrasound imaging. Field II [5] [6] is a well-known simulation software using linear acoustics, which is capable of calculating the emitted and pulse-echo fields for both the pulsed and continuous wave case for a large number of different transducers. For simulating non-linear ultrasound transducer fields and non-linear ultrasound imaging, the extension of Field II has to be provided with the capability of calculating non-linear pulse-echo fields.

An angular spectrum approach (ASA) that solves the nonlinear Westervelt wave equation [7] is selected to expand Field II to the non-linear simulation. The first step in attaining this is to develop the ASA to a linear pulsed simulation and validate it against results from Field II. This is the topic of this paper.

\section{THEORY}

\section{A. Conventional ASA}

Conventional ASA is a technique for monochromatic modeling of the propagating wave field. A two dimensional plane in space is used as the wave source. In the simulation it is usually parallel to the surface of the ultrasound transducer. Once the source is determined, the pressure in planes parallel to the source can be simulated by taking into account the propagation distance as shown in Fig. 1. $P_{0}$ is the sound pressure at the source plane (solid square), which is generated by the ultrasound probe on the top. The simulated plane (dashed square) $P_{n}$ along the propagation distance ( $z$-direction in the Cartesian coordinate) can be at any position. This is formulated as [8] [9]

$$
P_{n}\left(x, y, z_{n}\right)=\mathbf{F}^{-1}\left\{\hat{P}_{0}\left(k_{x}, k_{y}, z_{0}\right) e^{-j k_{z}\left(z_{n}-z_{0}\right)}\right\},
$$

where the subscript variable $n$ is an integer number meaning any arbitrary propagation distance along the $z$ direction, $\mathbf{F}^{-1}\{\}$ represents the inverse Fourier transform, and $\hat{P}_{0}\left(k_{x}, k_{y}, z_{0}\right)$ is a $2 \mathrm{D}$ spatial Fourier transform of the source pressure given by

$$
\hat{P}_{0}\left(k_{x}, k_{y}, z_{0}\right)=\iint P_{0}\left(x, y, z_{0}\right) e^{-j\left(k_{x} x+k_{y} y\right)} d x d y .
$$

$k_{x}, k_{y}$ and $k_{z}$ are the wave numbers along the respective axes, and $k_{z}$ can be obtained by

$$
k_{z}= \pm \sqrt{k^{2}-k_{x}^{2}-k_{y}^{2}}
$$

where $k=\omega / c$ is the angular frequency $\omega$ divided by the sound speed $c$. 


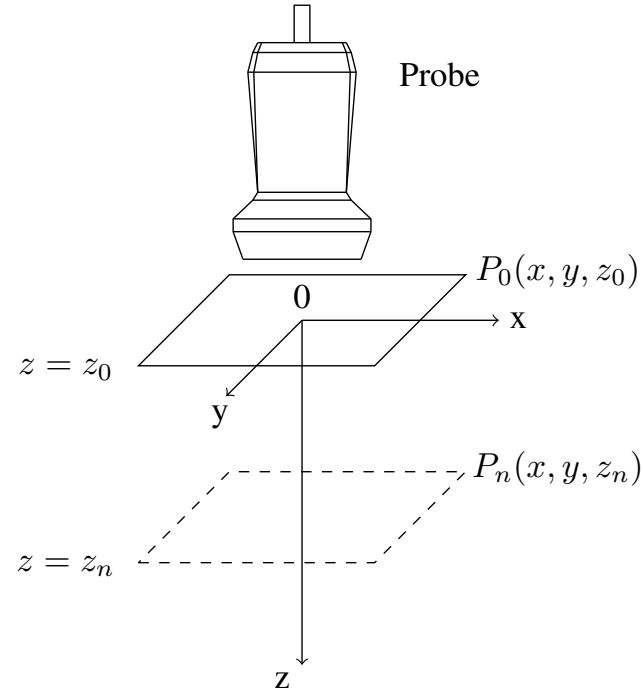

Fig. 1: Angular spectrum simulation for the wave propagation.

\section{B. Pulsed ASA}

The conventional ASA is calculated for a single frequency. The source pressure $P_{0}$ is obtained by the temporal Fourier transform. Only one frequency component is extracted, such as the fundamental frequency component or the mean value over all frequency components, that is a solely spatial-dependent value. To simulate the pulsed fields, the modified source pressure is supposed to be 4D-based like $P_{0}\left(x, y, z_{0}, t\right)$. Thus, after applying a 1D temporal Fourier transform to the source pressure, each frequency component of $P_{0}\left(x, y, z_{0}, f\right)$ will be processed by the ASA separately and summed in phase after propagation. The wave number $k$ is not a constant any longer and depends on each temporal frequency satisfying $k=2 \pi f / c$. Therefore, in pulsed ASA, (1) should be modified to

$$
P_{n}\left(x, y, z_{n}, t\right)=\mathbf{F}_{3 D}^{-1}\left\{\hat{P}_{0}\left(k_{x}, k_{y}, z_{0}, f\right) e^{-j k_{z}\left(z_{n}-z_{0}\right)}\right\},
$$

accordingly, (2) is rewritten as

$$
\begin{aligned}
& \hat{P}_{0}\left(k_{x}, k_{y}, z_{0}, f\right)= \\
& \iiint P_{0}\left(x, y, z_{0}, t\right) e^{-j\left(k_{x} x+k_{y} y+2 \pi f t\right)} d t d x d y,
\end{aligned}
$$

where $\mathbf{F}_{3 D}^{-1}$ represents a three dimensional inverse Fourier transform (1D $t \leftrightarrow f$ for time, 2D $x, y \leftrightarrow k_{x}, k_{y}$ for space), and $k_{z}$ is derived from (3) with the variable $k$ corresponding to each frequency $f$.

\section{METHOD}

Implementation is made with reference to Field II, since the expansion of Field II to non-linear pulsed-echo fields is the final goal. In this study, Field II does two tasks. One is for generating the source pressure, and the propagation to the simulated plane is calculated by pulsed ASA. The other is as a reference for the pulsed ASA simulation.

A three dimensional matrix $P_{0}\left(x, y, z_{0}, t\right)$ (constant $z_{0}$ and variable $x, y, t)$ is generated by Field II as the source. After 3D Fourier transform by (5), $\hat{P}_{0}\left(k_{x}, k_{y}, z_{0}, f\right)$ is applied to create

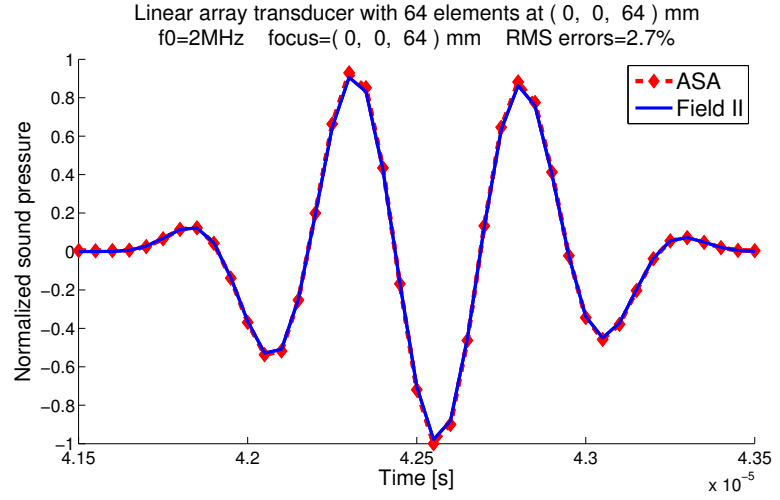

Fig. 2: Time response in the focus point at $(0,0,64) \mathrm{mm}$ for a linear array transducer, with reference to Field II.

$P_{n}\left(x, y, z_{n}, t\right)$ using (4). Finally, $P_{n}\left(x, y, z_{n}, t\right)$ is recalculated by Field II at the same location to validate the result made by pulsed ASA.

\section{RESUlts}

A linear array 64-element transducer with a center frequency of $2 \mathrm{MHz}$ is used in the simulation modeled by Field II. Each element has a $4 \mathrm{~mm}$ height and $0.7 \mathrm{~mm}$ width, and the kerf is $1 / 10$ of the width of the element, resulting in a total aperture of $4 \times 49.2 \mathrm{~mm}^{2}$. The source plane is $2 \mathrm{~mm}$ from the transducer surface, and the simulated plane is $64 \mathrm{~mm}$ from the aperture, the same as the electronic focus distance set in Field II. A rectangular plane with $33 \times 407$ points in space is selected as the source plane which covering an area of $12.7 \times 156.3 \mathrm{~mm}^{2}$. The spatial interval between adjacent points is $1 / 2$ wavelength that satisfies the Nyquist sampling rate.

The 2D beam profiles are simulated at $z=64 \mathrm{~mm}$ monochromatically, using the conventional ASA and Field II respectively, and shown with a dynamic range of $100 \mathrm{~dB}$ in Fig. 3. The pulsed ASA time response at $(0,0,64) \mathrm{mm}$ is illustrated in Fig. 2. The root mean square (RMS) error is calculated using Field II as a reference given by

$$
R M S \text { error }=\sqrt{\frac{\sum(A S A-F i e l d I I)^{2}}{\Sigma F i e l d I I^{2}}},
$$

where the variables $A S A$ and FieldII are the discrete matrix results calculated by pulsed ASA and Field II respectively. For the further validation, the time responses of 3 randomly selected points in the simulated plane are plotted in Fig. 4. The RMS error for all the points in the entire plane is $10.9 \%$ relative to the results of Field II. The calculation time for the ASA simulation of the pulses for all the points in one plane is less than 5 min with a 4-core $2.4 \mathrm{GHz}$ CPU and $4 \mathrm{~GB}$ memory computer using Matlab.

\section{Discussions}

The time response at the focus point displayed in Fig. 2 is very accurate, as the RMS error is only $2.7 \%$. Fig. 4 shows that the RMS error is increased, when the point of the simulated pulse is far from the focus center, but the curves still look matched even though the amplitudes are small. Fig. 6 displays the 


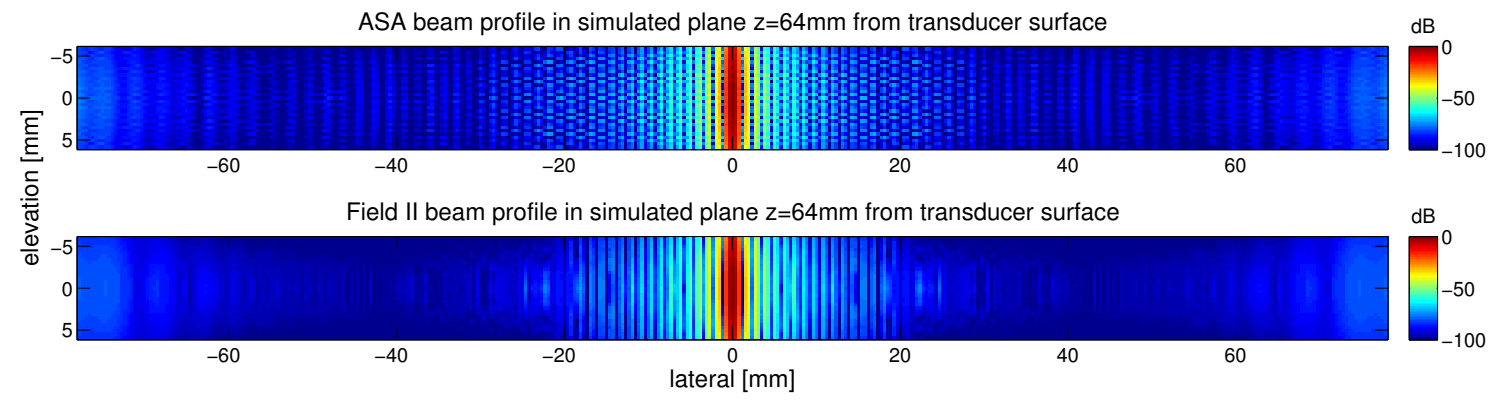

Fig. 3: Comparison of monochromatic beam profiles between ASA and Field II at the transducer electronic focus distance $z=64 \mathrm{~mm}$.

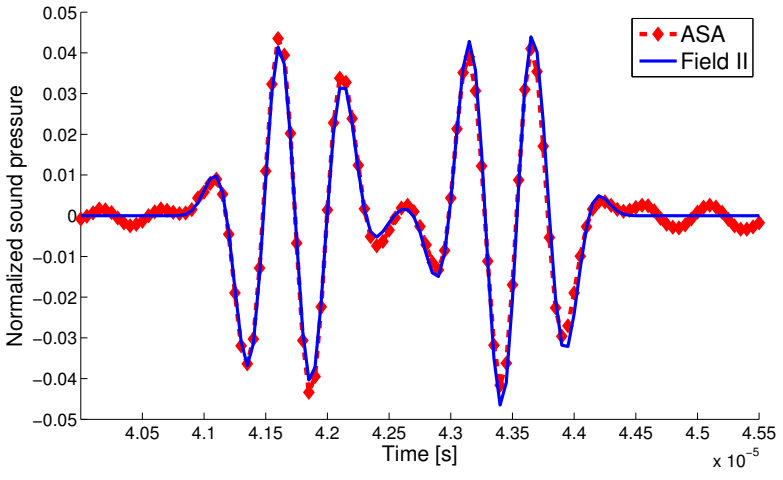

(a) RMS error $=12.5 \%$ at $(3.9,-1.5,64) \mathrm{mm}$

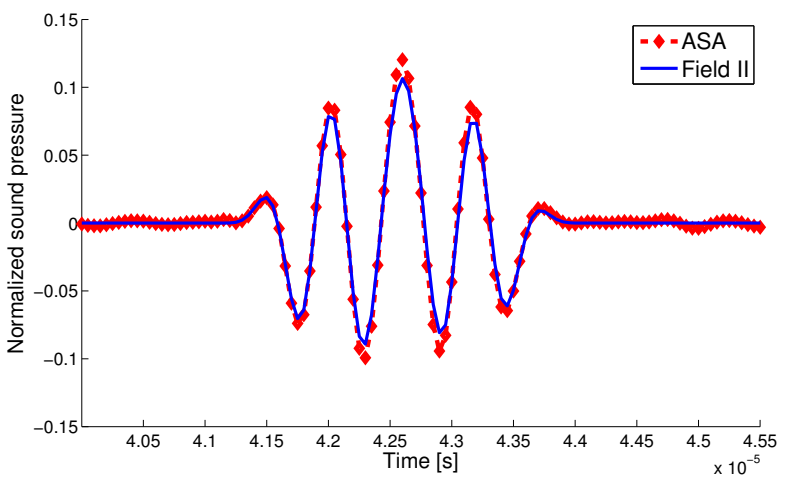

(b) RMS error $=13.3 \%$ at $(-1.9,1.9,64) \mathrm{mm}$

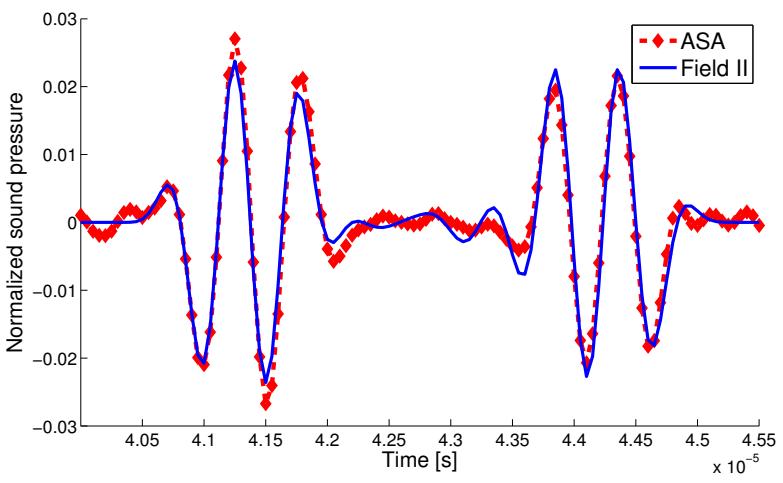

(c) RMS error $=23.4 \%$ at $(6.2,-4.2,64) \mathrm{mm}$

Fig. 4: Three comparisons of the time responses in three different positions between pulsed signals generated by ASA and Field II.
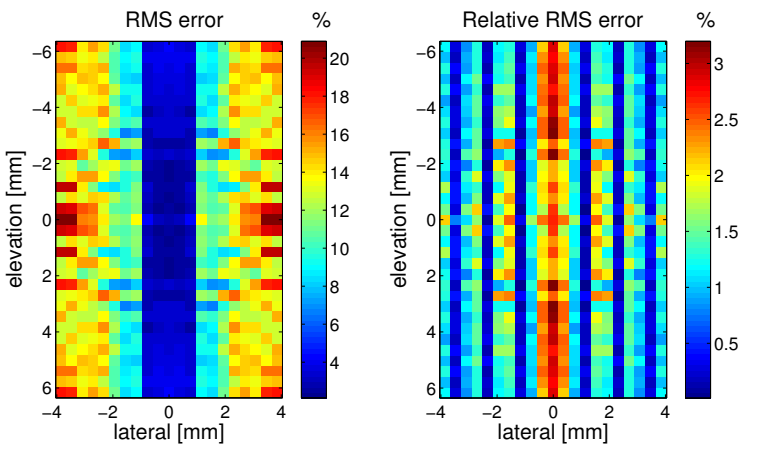

Fig. 5: RMS error vs Relative RMS error in the simulated plane.

distribution of RMS error of each pulse corresponding to the spatial point in the simulated plane. In connection with Fig. 3, it can be seen that the magnitudes of the signals from $\pm 20 \mathrm{~mm}$ to $\pm 50 \mathrm{~mm}$ in the lateral direction are small and thereby can be ignored though there are large errors (approximately 100\%). To study the errors in the focus area, the error distribution is shown in Fig. 5. The relative RMS error (Relative RMS error $=R M S$ error $\times$ Normalized sound pressure $)$ is used to express the true influence of the errors. The central line of the RMS error together with the normalized sound pressure in the simulated plane are plotted in Fig. 7a, which shows that the large errors will not have a strong impact on the results, due to the small amplitude of the sound pressure at the corresponding location. This can be further validated by Figs. $7 \mathrm{~b}$ and $7 \mathrm{c}$ at the different propagating distances $z=36 \mathrm{~mm}$ and $z=90 \mathrm{~mm}$ from the transducer surface. The relative RMS error curves are plotted in red at the bottom of each graph in Fig. 7. To show the potential of pulsed ASA, a phased array transducer is also modeled using the width of less than $1 / 2$ wavelength for each element to avoid the grating lobes, and the time response at the focus point referring to Field II is illustrated in Fig. 8. The good agreement is also seen even if the focal point is not in the center.

\section{CONCLUSIONS}

The theory of pulsed ASA is demonstrated in this study, which has been validated against Field II simulation. The plots of the time response for each single point in space show a good 


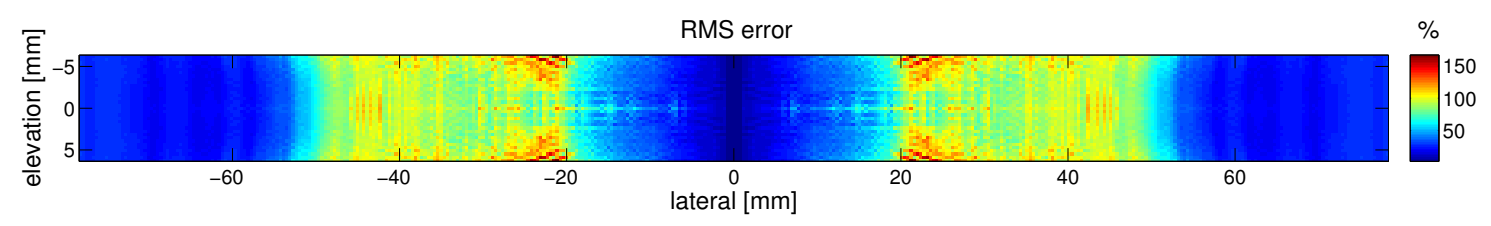

Fig. 6: RMS error distribution in the simulated plane with reference to Field II.

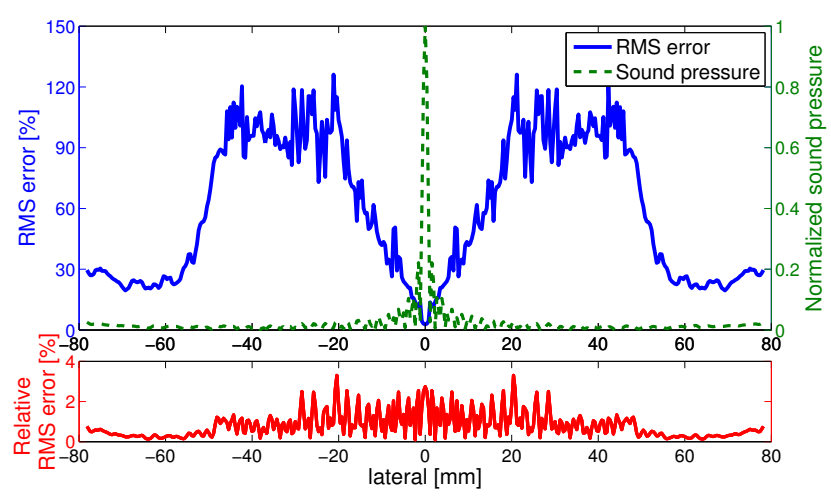

(a) $\mathrm{z}=64 \mathrm{~mm}$

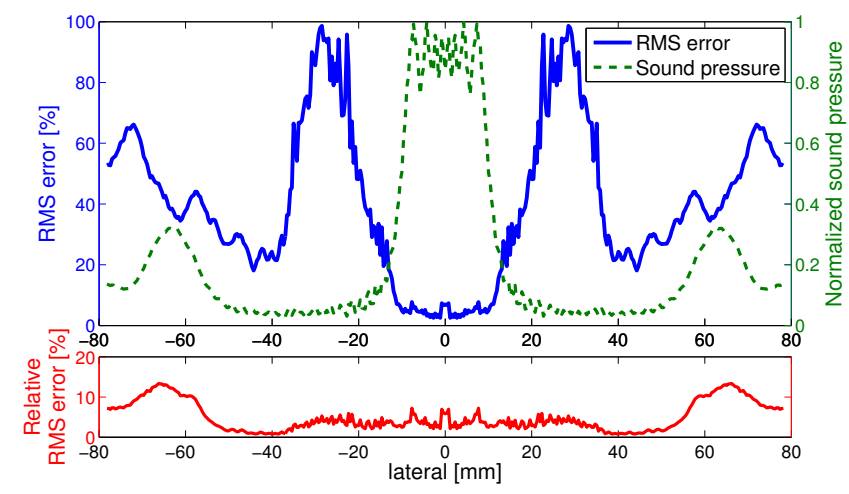

(b) $\mathrm{z}=36 \mathrm{~mm}$

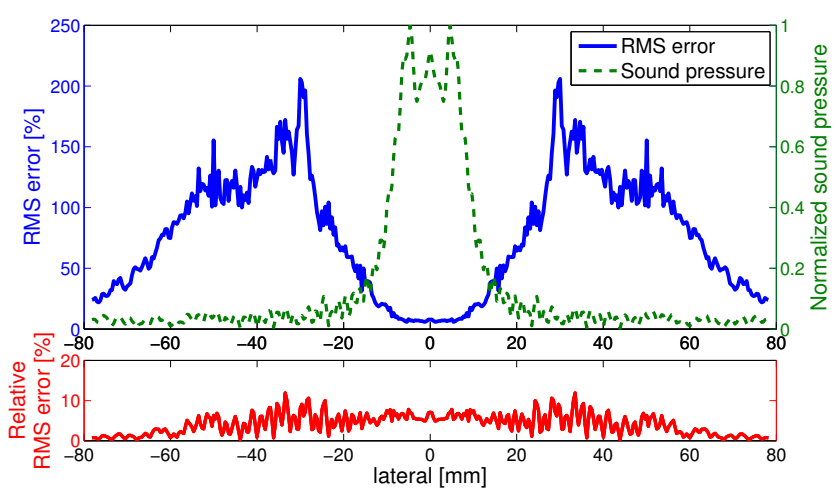

(c) $\mathrm{z}=90 \mathrm{~mm}$

Fig. 7: RMS (blue) and relative RMS (red) errors distributions corresponding to the magnitude of sound pressure (green) at different propagating distances, the simulated planes are for (a) $64 \mathrm{~mm}$, for (b) $36 \mathrm{~mm}$ and for (c) $90 \mathrm{~mm}$ from the transducer surface respectively.

agreement between the simulations made by pulsed ASA and by Field II. The analysis of the distribution of RMS errors

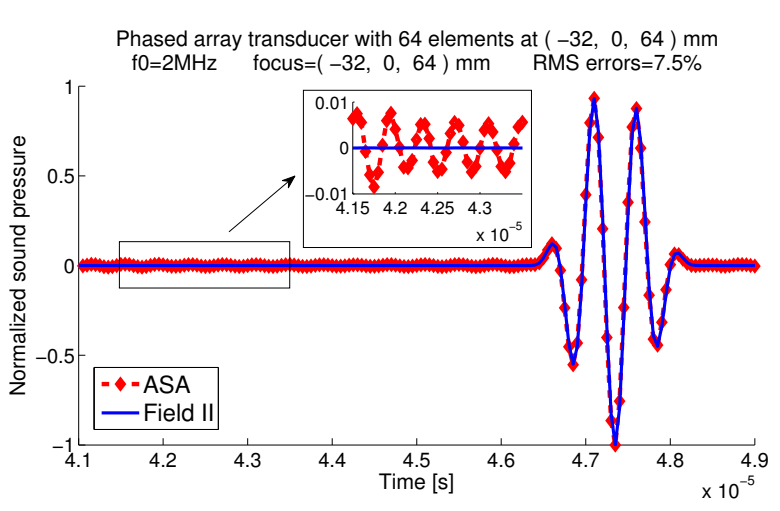

Fig. 8: Time response in the focus point at $(-32,0,64) \mathrm{mm}$ for a phased array transducer, with reference to Field II.

shows that the accuracy of the time response at each position in the whole simulated plane is acceptable. The creation of linear pulsed ultrasound field by the use of pulsed ASA is the first step in the extension of Field II to simulate non-linear pulsed fields for ultrasound imaging.

\section{ACKNOWLEDGMENT}

This work was supported by grant 26-04-0024 from the Danish Science Foundation, Technical University of Denmark, and by BK Medical Aps.

\section{REFERENCES}

[1] S. Holm, "Ultrasim - a toolbox for ultrasound field simulation," in Nordic Matlab conference, 2001.

[2] B. Piwakowski and B. Delannoy, "Method for computing spatial pulse response: Time-domain approach," in J. Acoust. Soc. Am., Dec. 1989, vol. 86, pp. 2422-32.

[3] R. O. Cleveland and M. F. Hamilton, "Time-domain modeling of finiteamplitude sound in relaxing fluids," in J. Acoust. Soc. Am., 1996, vol. 99, pp. 3312-18.

[4] Y. S. Lee and M. F. Hamilton, "Time-domain modeling of pulsed finiteamplitude sound beams," in J. Acoust. Soc. Am., 1995, vol. 97, pp 906-917.

[5] J. A. Jensen and N. B. Svendsen, "Calculation of pressure fields from arbitrarily shaped, apodized, and excited ultrasound transducers," IEEE Trans. Ultrason., Ferroelec., Freq. Contr,, vol. 39, no. 2, March 1992.

[6] J. A. Jensen, "Field: A program for simulating ultrasound systems," in Medical \& Biological Engineering \& Computing, 1996, vol. 34, pp. 351353.

[7] Y. Xiang and M. F. Hamilton, "Angular spectrum decomposition analysis of second harmonic ultrasound propagation and its relation to tissue harmonic imaging," NDE, June 192006.

[8] Y. Du and J. A. Jensen, "Feasibility of non-linear simulation for Field II using an angular spectrum approach," in 2008 IEEE Ultrason. Symp.,, 2008, pp. 1314-1317.

[9] J. W. Goodman, Introduction to Fourier Optics, McGraw-Hill, New York, 1996. 